\title{
Performance evaluation of Jaipur knee joint through kinematics gait symmetry with unilateral transfemoral Indian amputees
}

\author{
Pawan Mishra ${ }^{1}$, Sachin Singh ${ }^{2}$, Vinayak Ranjan ${ }^{3}$, Sonu Singh ${ }^{4}$, Anuradha Pandey ${ }^{5}$, \\ Madhusmita Mohanta ${ }^{6}$, Deepali Atheaya ${ }^{7}$ \\ ${ }^{1,2}$ Indian Institute of Technology (IIT-ISM), Dhanbad, India \\ ${ }^{3,6,7}$ Bennett University, Greater Noida, India \\ ${ }^{4}$ Army Hospital, R\&R, New Delhi, India \\ ${ }^{5}$ Gautam Buddha University, Greater Noida, India \\ ${ }^{1}$ Corresponding author \\ E-mail: ${ }^{1}$ pawan.mishra@bennett.edu.in, ${ }^{2}$ sachinsingh47@gmail.com, ${ }^{3}$ vinayak.ranjan@bennett.edu.in, \\ ${ }^{4}$ sonusingh76@rediffmail.com, ${ }^{5}$ anu.pandey@gmail.com,6mm1552@bennett.edu.in, \\ ${ }^{7}$ deepali.atheaya@bennett.edu.in
}

Received 18 November 2018; accepted 26 November 2018 DOI https://doi.org/10.21595/vp.2018.20398

Check for updates

Copyright $(2018$ Pawan Mishra, et al. This is an open access article distributed under the Creative Commons Attribution License, which permits unrestricted use, distribution, and reproduction in any medium, provided the original work is properly cited.

\begin{abstract}
Human gait data becomes very helpful in the prediction and correction of movement control while walking. In many areas like medical practices, Physical fitness training programs, rehabilitation techniques and prosthesis performance, gait study has its significant scientific relevance and applications. This study aims to predict the performance of Jaipur knee joint in terms of gait symmetry with transfemoral Indian amputees. First time we tried to calculate gait symmetry of widely used Jaipur knee joint with Indian population. This aspect has not been focused so far with Indian amputees. 11 unilateral transfemoral amputees $(9$ men and 2 women; average age 45 years, range 31-58 years) participated in the study. Subjects were using prosthesis with Jaipur knee for long time (mean: 16 yrs; range: 5-27 yrs). To investigate the gait function with 6 Vicon cameras 3D motion analysis system (Kinematrix system) participants were asked to walk with their comfortable speed. Gait symmetry, might be the basis of recommendation of knee joint. Kinematics parameter is predicted quantitavely gait symmetry with Jaipur knee joint. This research will result in significant prevention of degenerated musculoskeletal effects in future generally seen in unilateral transfemoral amputees.
\end{abstract}

Keywords: amputation, gait, Jaipur knee, symmetry, transfemoral.

\section{Introduction}

Human gait data becomes very helpful in the prediction and correction of movement control while walking. In the areas like medical practices, physical fitness training programs, rehabilitation techniques and prosthesis performance, gait study has its significant scientific relevance and applications. In these images are gathered and recognized as per positions of reflective markers on pelvis, hip, knee and ankle joints [1]. Balzac considered 'Theory of walking' [2], gait analytical observation [3]. The metric discussed by Ramkrishnan et al. served as a significant tool to classify and distinguish between multiple asymmetric bi pedal gaits [4].

Bhagwan Mahaveer Viklang Sahayta Samiti (BMVSS) team, who made 'Jaipur foot' and Stanford university students developed Jaipur knee as joint efforts. This joint is manufactured in India which is recognized as one of the top 50 best inventions by Time magazine in the year 2009 [5]. BMVSS has successfully fitted more than 9000 knee joints through its no cost license. This would be also easy for clinicians to recommended suitable knee joint on the basis of kinematics data symmetry [6]. This study aims to quantify the performance of Jaipur knee joint in case of transfemoral Indian amputees.

The gait asymmetry is generally observed in case of unilateral transfemoral amputees [7]. 
Non-uniform distribution of load may result to pain experienced in back and in natural leg [8,9] and other musculoskeletal degenerative problems [10]. Jaroslav et al. determined the effect of knee joint type which used on their symmetry and compared pelvic movements in transfemoral amputees by using different types of knee joints [11].

The gait asymmetry is assessed by symmetry index (SI) calculation [12], a ratio index (RI) [13] or a symmetry angle (SA). In the gait cycle report all these indices are discussed in context of single point in gait cycle and thus limit the final assessment. The variation among the data noted in performance of both limbs is gait asymmetry and it has also been discussed with the use of MANOVA, $t$-test tool and variance ratio, principal component analysis [14], correlation coefficients, coefficients of variation, root mean-square (RMS) difference methods and cross-correlation techniques [15]. In this study, participants walked on flat pathway and we measured the effect of Jaipur knee with kinematics parameters. Al-Fatafta et al. examined at the test and re-test repeatability of knee joint kinematics in the sagittal; frontal; transverse planes during stair climbing with measurement error calculation [16].

\section{Methodology}

\subsection{Participants}

The eleven participants (Table 1) suffering from above knee amputation in one limb after a physical injury or trauma, with no comorbidity voluntarily are involved in this study. On prior to study, written consent letter was taken from all participants. Every one of them was used to wear prosthesis for many years. During the experiment, the same patients were asked to wear their prosthesis with Jaipur knee for a certain acclimation period suggested by the clinician. The research was carried under the supervision of trained and skilled staff at gait lab. All the steps to be involved in this study were discussed with subjects. Gait lab staff verified for comfortable socket fit, the overall fitment and alignment of prosthesis to ensure stability and comfort for amputees. A complete physical checkup of all participants, was done based on their history and current data of back joints, hip joints and knee joint, any prior disease or injury etc. This pre-assessment of experienced clinician was to ensure that no related issues exist which may have altered their walking pattern. Improper alignment may result in uncomfortable fit and non-uniform pressure distribution on socket limb joint boundary and it may lead to reduced comfort, uneasiness and potential tissue injury [17].

Table 1. Details of participants with above knee amputation in one leg (Abbreviation: PVD - peripheral vascular disease; CA - cancer)

\begin{tabular}{|c|c|c|c|c|c|c|}
\hline Participants & Age (years) & Sex & Causes & $\begin{array}{c}\text { Amputation } \\
\text { period (years) }\end{array}$ & Prosthetic foot & $\begin{array}{c}\text { Acclimation } \\
\text { Period (weeks) }\end{array}$ \\
\hline A & 26 & M & Trauma & 12 & Jaipur foot & 12.4 \\
\hline B & 44 & M & Trauma & 17 & Jaipur foot & 13.7 \\
\hline C & 58 & M & PVD & 19 & Jaipur foot & 13.2 \\
\hline D & 42 & M & Trauma & 15 & Jaipur foot & 14.9 \\
\hline E & 38 & F & Trauma & 10 & Jaipur foot & 10.6 \\
\hline F & 60 & M & CA & 29 & Jaipur foot & 14.9 \\
\hline G & 39 & M & Trauma & 7 & Jaipur foot & 11.7 \\
\hline H & 47 & F & Trauma & 13 & Jaipur foot & 12.5 \\
\hline
\end{tabular}

\subsection{Gait analysis}

For kinematic evaluation data were recorded with the help of computerized video motion analysis system which included six Vicon camera having a frame rate of 60 (frames per seconds) fps and using infrared (IR) light-emitting diode strobes. The cameras were located such that they yielded accurate movement data of prominent joints like hip joints, knee joint and ankle joint for 
both legs in sagittal plane. The reflective markers were located to setup anatomic coordinate system at prescribed locations for gait test. Then movement data of both legs were recorded and processed by the motion capture system. During each trial first static data was recorded when participant was standing comfortably. This data supported in calculations of joint centers positions. A strain-gauzed AMTI force plate dimensions $(508 \mathrm{~mm} \times 460 \mathrm{~mm})$ was fitted on midpoint of defined pathway, measured data for ground reaction forces. The force plate data was synchronized to the computer system. The data received from marker coordinate system and from AMTI force plate were acted as input to calculate kinematics data. Two consecutive heel strike points were considered as gait cycle. Intermediate states of gait were represented as percentage of total distance during gait.

\subsection{Symmetry index}

The kinematics performance of both knee joints in comparison with intact leg is measured with symmetry index. The main idea to calculate symmetry was to evaluate notable distinctions by quantitative approach between the both legs during gait like Robinson adopted algebraic method to calculate symmetry index [18]:

$S I=\frac{2\left(X_{R}-X_{L}\right)}{\left(X_{R}+X_{L}\right)} \times 100 \%$

where $X_{R}$ and $X_{L}$ are gait variables for both legs respectively. Ideally symmetry index should be equal to zero, when calculated the all gait variables on discrete level. The approach used in this study was reported by Crankshaw [19], he utilized singular value decomposition method. As acquired data was in a waveform. All variables got subtracted from its average values and translated accordingly. The resulted new data point were calculated as $2 \times n$ matrix $M$. Eigen values along with Eigen vectors were determined from the matrix $M$. In graph, ' 0 ' value indicates that the waveform was not affected while +1 and -1 donated the points of model symmetry and model asymmetry respectively. This data acquisition was done for both legs and kinematic analysis was done for hip joint, knee joint and ankle joints.

The movement of joints have been captured in sagittal plane. For statistics, a standard statistical analysis tool was used. As discussed before that two gait phases with one knee joints were recorded in many trials therefore two way analysis of variance (ANOVA) approach was preferred to measure deviation of symmetry while using two different knee joints units in prosthesis. Statistical significance of $p \leq 0.05$ was considered.

\section{Result}

\subsection{Participants}

In this research, 11 unilateral transfemoral subjects [ 9 men and 2 women; mean age 45 years, $\left(\mathrm{SD}=12\right.$ years,31-58 years range); and mean body mass index $(\mathrm{BMI})$ of $29 \mathrm{~kg} / \mathrm{m}^{2},\left(\mathrm{SD}=6 \mathrm{~kg} / \mathrm{m}^{2}\right)$ were participated. These participants were used to wear prosthesis for long time with mean data of 16 years. They were tested with prosthesis with Jaipur knee and re-examined with an acclimation period (Average 14 weeks, $\mathrm{SD}=6$ weeks).

\subsection{Kinematic symmetry}

The symmetry as shown in (Fig. 1) with kinematic data is different as found by gait phase and joint level. The joints movement have recorded in sagittal plane, it is evident that appreciable symmetry is shown in swing phase as well as in stance phase of gait cycle. Index reported symmetry more than 0.90 . In stance and swing phases, the sagittal motion exhibited good 
symmetry where the symmetry index was more than 0.90 at hip joint in the amputee population, standard significance was set $(p=0.14)$. At knee joint notable symmetry deviation was seen during swing phase and stance phase $(p=0.006)$ indicating maximum deviation in stance phase of knee joint. There was least symmetry reported during stance phase on changing knee locations with respect to loading conditions. On contrary many participants justified kinematics symmetry during swing phase $(p=0.36)$. Likewise, at hip joint and ankle joint also, appreciable symmetry was reported in swing and stance phase. Data reported maximum asymmetry in graph indexed near to -1 . In above knee prosthesis foot unit experiences less planter flexion than intact leg this causes maximum asymmetry at ankle joint in swing phase. The data from a representative subject may justify logically the visible in symmetry obtained in Jaipur knee (Fig. 2). The participants reported increased knee flexion against loading during gait, this response appreciably got compensated. That is why knee joint deviation in stance reported significant -0.012 .

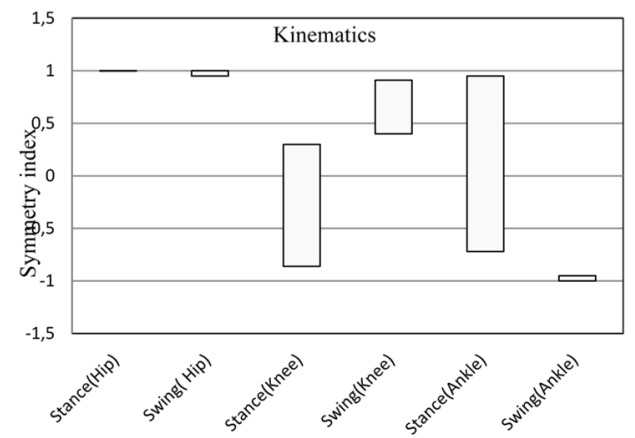

Fig. 1. Kinematics symmetry index for joints (hip, knee, ankle) resulted from Jaipur knee joint recorded in sagittal plane

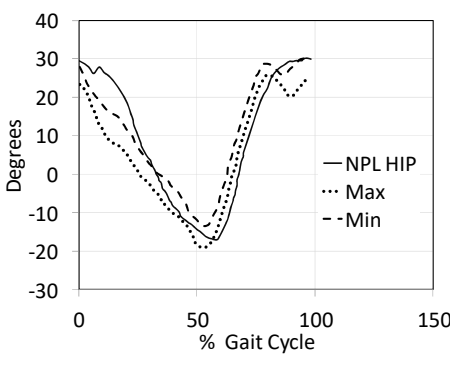

a)

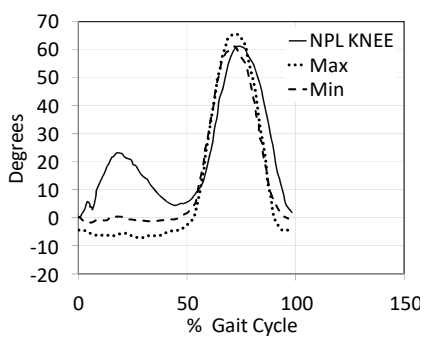

b)

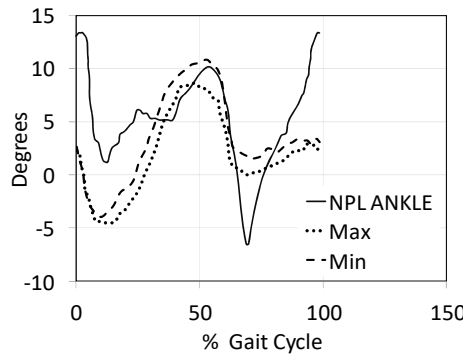

c)

Fig. 2. Kinematics results in sagittal plane indicating hip, knee $\&$ ankle joints for non-prosthetic leg NPL (solid line) versus the prosthetic leg (dashed line) where the participants were using a Jaipur knee joint.

Table 2. Details of participants with above knee amputation in one leg (Abbreviation: PVD - peripheral vascular disease; CA - cancer)

\begin{tabular}{|c|c|c|c|c|c|c|c|c|c|c|c|}
\hline \multicolumn{4}{|c|}{ Hip } & \multicolumn{4}{c|}{ Knee } & \multicolumn{4}{c|}{ Ankle } \\
\hline \multicolumn{2}{|c|}{ Stance } & \multicolumn{2}{c|}{ Swing } & \multicolumn{2}{c|}{ Stance } & \multicolumn{2}{c|}{ Swing } & \multicolumn{2}{c|}{ Stance } & \multicolumn{2}{c|}{ Swing } \\
\hline Max & Min & Max & Min & Max & Min & Max & Min & Max & Min & Max & Min \\
\hline 0.996 & 0.989 & 0.991 & 0.985 & 0.356 & -0.01 & 0.947 & 0.952 & 0.711 & 0.748 & -0.99 & 0.991 \\
\hline
\end{tabular}

\section{Discussion}

The objective of this research was to evaluate and quantify the performance of Jaipur knee joint in terms of symmetry in gait cycle. For this participants used above knee prosthesis with Jaipur knee joint. To compare with ease, a healthy person's gait cycle may be assumed as perfect symmetry. To compare the performance of these knee joints Kauffman's technique was adopted. 
Kauffman did not only compare the points parallel to gait cycle but also utilized complete wave-front geometry [20]. The outcome indicated almost similar kinematics data at hip, knee and ankle joints, when different participants used Jaipur knee joint. Moreover, maximum flexion range at knee joint observed with better reduction in swing phase with Jaipur knee joints. When foot rests on ground during walk, knee joint ensures stability hence visibly enhanced moment at knee joint is clinically very significant. The outcome of research supports the fact that Jaipur knee joint enhances the symmetry during gait as compared to intact leg and this will ensure better balance and sound stability.

Besides this above findings, few limitations were comprised with this study like first was testing orders in prosthesis were not random. Participants first used prosthesis with different knee joints then they were asked to wear prosthesis with Jaipur knee joints. It may be considered conventional clinical context for the amputees on Jaipur joint. Second may be participants' walking speed was as per their comfort and were different with that of one another. Lelas et.al. discussed that different walking speeds may result in according differences in data used to calculate kinematics related to walking speed [21]. Likewise Nolan et.al. concluded the effects seen in gait symmetry of participants due to different walking speed [22]. Our result could have affected due to change in walking speed. However, in this study the variation of walking speeds was very small (approximate $0.06 \mathrm{~m} / \mathrm{s}$ ) and such small gap will not considerably affect the results of this study.

\section{Conclusions}

On the basis of outcomes of this research, when participants with above knee amputation in one leg walked on pathway with the speed as per their comfort and ease with Jaipur knee joints in prosthesis, they demonstrated appreciably reduced asymmetry. This conclusion quantified and measured differences in participants with Jaipur joint, this study will be useful for clinicians as they will find quantification of observed data like symmetry and can better predict about response and performance of the prosthetic with different knee joints. This study will also be useful for the amputees during assessment and selection of knee joint to avoid future disorders due to poor performance of the knee joint.

\section{References}

[1] Hodgins D. The importance of measuring human gait. Medical Device Technology, Vol. 19, Issue 5, 2008, p. 44-47.

[2] Balzac H. D. Theorie de la Demarche. Didier, Paris, 1853.

[3] Collado Vazqueza S., Carrillo J. Balzac and human gait analysis. Neurologia, Vol. 30, Issue 4, 2015, p. 240-246.

[4] Ramakrishnan T., Lahiff C., Reed K. B. Comparing gait with multiple physical asymmetries using consolidated metrices. Frontoers in Nerorobotics, Vol. 12, Issue 2, 2018.

[5] Time Magazine. 2009, htp://content.time.com/time/specials/packages/article/0,28804,1934027 1934003 1933963,00.html.

[6] Bhagwan Mahavir Sahayta Samiti. Jaipur, 2016, http://jaipurfoot.org.

[7] Jaegers S. M. H. J., Arendzen J. H., De Jongh H. J. The prosthetic gait of unilateral transfemoral amputees: a kinematic study. Archives of Physical Medicine and Rehabilitation, Vol. 76, Issue 8, 1995, p. $736-743$.

[8] Bautmans I., Jansen B., Van Keymolen B., Mets T. Reliability and clinical correlates of 3D-accelerometry based gait analysis outcomes according to age and fall-risk. Gait and Posture, Vol. 33, 2011, p. 366-372.

[9] Kulkarni J., Adams J., Thomas E., Silman A. Association between amputation, arthritis and osteopenia in British male war veterans with major lower limb amputations. Clinical Rehabilitation, Vol. 12, 1998, p. 348-353. 
[10] Johansson J., Sherrill D., Riley P., Bonato P., Herr H. A clinical comparison of variable damping and mechanically passive prosthetic knee devices. American Journal of Physical Medicine and Rehabilitation, Vol. 84, 2005, p. 563-575.

[11] Jaroslav U., Jandacka D., Farana R., Zahradnik D., Rosicky J., Janura M. Kinematics of gait using bionic and hydraulic knee joints in transfemoral amputees. Acta Gymnica, Vol. 47, Issue 3, 2017, p. 130-137.

[12] Robinson R., Herzog W., Nigg B. Use of force platform variables to quantify the effects of chiropractic manipulation on gait symmetry. Manipulative Physiotherapy, Vol. 10, 1987, p. 172-176.

[13] Zifchock R., Davis I., Higginson J. R. T. Asymmetry angle: a novel, robust method of quantifying asymmetry. Gait and Posture, Vol. 27, 2008, p. 622-627.

[14] Sadeghi H. Local or global asymmetry in gait of people without impairments. Gait and Posture, Vol. 17, 2003, p. 197-204.

[15] Haddad J., Van Emmerik R., Whittlesey S., Hamill J. Adaptions in interlimb and intralimb coordination to asymmetrical loading in human walking. Gait and Posture, Vol. 23, 2006, p. 429-434.

[16] Al Fatafta H., Liu A., Hutchins S., Jones R. Knee joint kinematics and kinetics during the force plate attachable interlaced stairway climbing (FPAIS) in healthy individuals. Prosthetics and Orthotics Open Journal, 2017.

[17] Yang L., Solomonidis S., Spence W. P. J. The influence of limb alignment on the gait of above knee amputees. Journal of Biomechanics, Vol. 24, 1991, p. 981-997.

[18] Blumentritt S. A new biomechanical method for determination of static prosthetic alignment. Prosthetics and Orthotics International, Vol. 21, 1997, p. 107-113.

[19] Crenshaw S., Richards J. A method for analyzing joint symmetry abnormally with an application to analyzing gait. Gait and Posture, Vol. 24, 2006, p. 515-521.

[20] Kaufman K., Levine J., Brey R., Mccrady S., Padgett D., Joyner M. Energy expenditure and activity of transfemoral amputees using mechanical and microprocessor controlled prosthetic knees. Archieves of Physical Medicines and Rehabilitations, Vol. 87, 2008, p. 1382-1385.

[21] Lelas J., Merriman G., Riley P., Kerrigan D. Predicting peak kinematic and kinetic parameters from gait speed. Gait and Posture, Vol. 17, 2003, p. 106-112.

[22] Nolan L., Wit A., Dudzinski K., Lees A., Lake M., Wychowansky M. Adjustments in gait symmetry with walking speed in trans-femoral and trans-tibial amputees. Gait and Posture, Vol. 17, 2003, p. 142-151.

[23] Michael J. Modern Prosthetic knee mechanism. Clinical Orthopaedics Related Research, Vol. 361, 1999, p. 39-47.

[24] Richard V., Lamberto G., Lu T. W., Cappozzo A., Dumas R. Knee kinematics estimation using multi-body optimisation embedding a knee joint stiffness matrix: a feasible study. Plos One, Vol. 11, Issue 6, 2016, p. 1-18.

[25] Nicholas R. H., Rafferty D., Meleesa F. W., Simonson A., Lovalekar M., Reinert A., Sell T. Lending kinematics and kinetics at the knee during different landing tasks. Journal of Athletic Training, Vol. 52, Issue 12, 2017, p. 1101-1108.

[26] Maria S., Pablo C. Human gait kinematics measurement. Open Journal of Orthopedics, Vol. 7, 2017, p. 79-89. 